\title{
A qualitative study of online support communities for lung cancer survivors on targeted therapies
}

\author{
Casey A. Walsh ${ }^{1,2}$ (D) Morhaf Al Achkar ${ }^{3}$ (D)
}

Received: 9 October 2020 / Accepted: 6 January 2021 / Published online: 18 January 2021

(C) The Author(s), under exclusive licence to Springer-Verlag GmbH, DE part of Springer Nature 2021

\begin{abstract}
Background Due to recent treatment advances, people who have non-small cell lung cancer with oncogenic alterations are an important new group of cancer survivors. Little is known about lung cancer online support communities. This research was guided by two primary questions: (1) How do these lung cancer survivors engage in online support communities? and (2) What are the psychological, social, and physical impacts of such engagement?

Methods Qualitative in-depth interviews were conducted with patients with advanced lung cancer $(N=40)$ to learn about their experiences with the illness. We used qualitative thematic analysis, inductive and deductive, as outlined by Carspecken. We adapted the framework for studying online communities developed by Zhang and colleagues to examine engagement with and impacts of involvement in online lung cancer support communities.

Results Participants described engaging in the online community through (1) initializing communication through asking questions or sharing resources, (2) responding to others comments or inquiries, or (3) simply observing/reading others posts. Participation had physical, psychological, or social impacts, with benefits (e.g., empowerment) and risks (e.g., feelings of jealousy or misinformation) in each domain. Participants used various strategies to mitigate negative impacts, such as distancing oneself as needed.

Conclusions Online lung cancer support communities provide support, camaraderie, and specialized health information. However, there are also risks of online engagement, such as social comparison or accessing misinformation. Understanding the utility of online support communities for lung cancer survivors on targeted therapies and further addressing their risks are urgent tasks, especially in the post-COVID era.
\end{abstract}

Keywords Lung cancer $\cdot$ Online support $\cdot$ ALK $\cdot$ EGFR $\cdot$ ROS1 $\cdot$ Qualitative research

\section{Background}

Cancer survivors are increasingly turning to online communities to connect with peers, access support, and exchange information. Despite increasing prevalence and usage, no common definition exists for online cancer support communities

Casey A. Walsh

cwalsh2@uw.edu

1 Department of Health Services, University of Washington, Seattle, WA, USA

2 Clinical Research Division, Fred Hutchinson Cancer Research Center, 1100 Fairview Ave N., Mail Stop D5-220, Seattle, WA 98109, USA

3 Department of Family Medicine, University of Washington School of Medicine, Seattle, WA, USA
[1]. These communities' structure and format vary greatly, including public and private communication modes and ranging from small, diagnosis-specific clinician-moderated communities to unmoderated public groups on popular social media platforms. Member characteristics, such as age, diagnosis, and gender, can influence discussion content and group dynamics [2]. Individuals can choose the frequency and duration of their engagement and the nature of their involvement (e.g., if and how often they post messages). The asynchronous communication in online communities enables individuals to access information incrementally and from multiple individuals versus information received simultaneously from one source, such as during a medical consultation [3]. Shared understanding and experiences within condition-specific online communities, especially among individuals with rare diagnoses, help foster a sense of community and belonging [4]. However, risks and possible harmful effects of participation include 
exposure to misinformation, privacy concerns, and emotional burdens [3]. The most commonly cited factors that influence the extent to which patients are active on the Internet are demographic characteristics, including age, gender, education level, and stage of illness [5]. Since personal characteristics influence the effects of participation in online communities, patient preferences should guide how social contact occurs during their cancer care trajectory [5].

For individuals living with highly stigmatized conditions such as lung cancer, connecting with others with shared experience and engaging in lung cancer treatment and research advocacy efforts may help to improve quality of life [6]. Smoking-related stigma can constrain communication and limit help-seeking [7]. Lung cancer is the leading cause of cancer death in the USA [8]. Targeted therapies are changing the treatment paradigm for lung cancer, providing hope for those with limited treatment options [9]. Lung cancer patients live with chronic uncertainty about their mortality and seek others they can relate to [10]. This uncertainty can lead to introspective struggles with establishing priorities, living with purpose, and being understood by others [10].

Cancer survivors also face the devastating health, social, financial, and psychological burdens of the coronavirus disease 2019 (COVID-19). Individuals may experience distress and social isolation due to challenges and safety concerns in accessing their usual support networks [11]. Patients have fewer contact points with their physicians, other patients (e.g., informal interactions in the waiting room), and face-toface support groups. Therefore, it is urgent to identify and optimize online cancer support communities.

There is mixed efficacy of online peer support for cancer patients [12]. Emerging research examines the behaviors of lung cancer survivors when using different social media platforms (e.g., Facebook, Twitter) [13]. In addition to emotional and informational support, an unanticipated category of spirital support was found uniquely in the lung cancer support group on Facebook. Twitter posts showed the most companionship support, reflecting the use of hashtags and as usergenerated signals of belonging [13]. Building upon this emerging research, we explored two research questions: (1) How do lung cancer survivors engage in online support communities? and (2) What are the psychological, social, and physical impact(s) of such engagement?

\section{Materials and methods}

\section{Study design}

This is a cross-sectional analysis of qualitative interview data. The principal investigator (MA) conducted in-depth qualitative interviews individually with patients with advanced lung cancer on targeted therapies to learn about their experiences with the illness. The principal investigator is a male, lung cancer survivor, member of the ROS1ders' group, and practicing primary care physician with expertise in qualitative health research. The co-author $(\mathrm{CW})$ is a female, licensed social work clinician and researcher with a focus in adolescent and young adult oncology.

\section{Study population}

Forty participants met the following inclusion criteria: (1) metastatic or advanced non-small cell lung cancer with one oncogenic alteration (EGFR, ALK, or ROS1); (2) well enough physically and psychologically to participate; (3) English proficiency; and (4) receiving medical care in the USA. Patients were identified using purposive sampling from oncogenspecific online lung cancer support groups: the EGFR Resisters, the ALK-Positive Facebook Group, and the ROS1ders. There are volunteer moderators to help safeguard the online space. These are closed groups; to join, persons must have lung cancer or be a caregiver for someone with lung cancer. Each group included a few hundred to a thousand or more participants globally. Detailed methods are included in previous publications [ [10]].

\section{Study procedures}

Participants could be interviewed by phone, videoconference, or in-person based on location and preference. After obtaining verbal consent, the principal investigator (MA) conducted the interviews between August 2018 and March 2019. The interviews were audio-recorded and transcribed verbatim. Participants were asked to share their journey with cancer starting with diagnosis and treatment and to elaborate on what they were doing to cope with the illness and its impacts. Since participants were recruited from online support groups, the topic emerged naturally as part of what people engaged in to cope with the illness. As the topic emerged, the interviewer inquired about the experience in more detail. Examples of interview prompts are included in Table 1. Participants received a $\$ 50$ gift card as a sign of appreciation.

\section{Analysis}

NVIVO 11 was used to store and manage the de-identified interview data. We adapted the framework for studying online communities by Zhang and colleagues [2] which includes the main categories of engagement and impacts of online communities (Fig. 1). We adapted the sub-categories of engagement to include initializing, responding, and keeping at a distance. The sub-categories of impacts include physical, psychologi$\mathrm{cal}$, and social domains. We added examination of the perceived risks and benefits in each domain. We drew upon the theory of communication work in cancer [14] in which social 


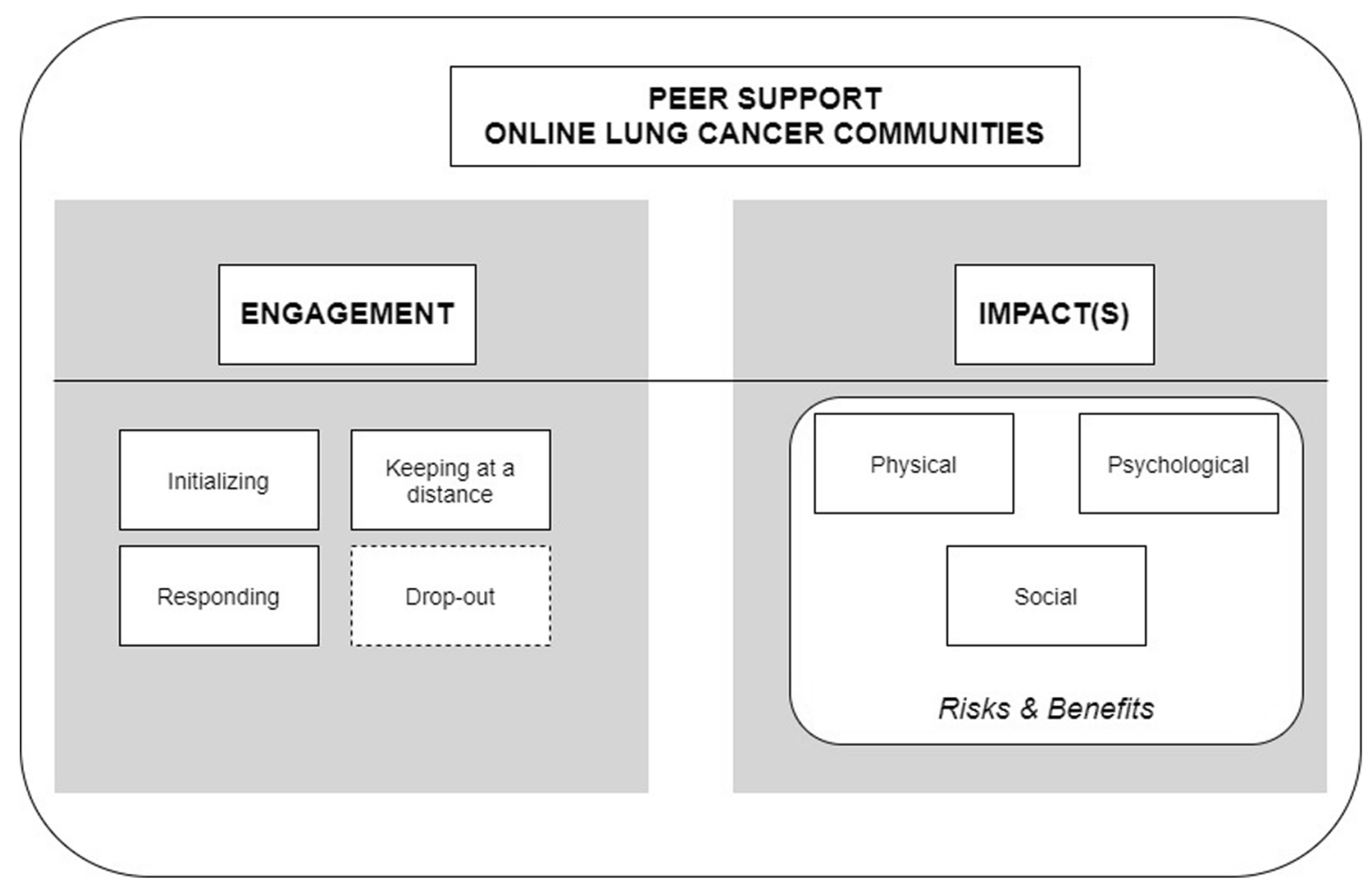

Fig. 1 Peer support in online lung cancer communities. We adapted the framework for studying online communities developed by Zhang et al. The dashed line represents that we did not have this data source in our study. We added analysis of the risks and benefits in each domain of impact

and information-related tasks inherent in coping with cancer can be laborious in and of themselves, and constitute a substantial and conceptually distinct line of work. The processes of exchanging messages are integral throughout cancer experience. We also drew upon the theory of "distributed health literacy" [15] in which people with chronic health conditions utilize their social network for support with health literacyrelated tasks such as managing their condition and making treatment decisions.

We used interview data from lung cancer patients on targeted therapies describing their perspectives about online lung cancer support groups on Facebook. We used deductive qualitative analysis, informed by the framework for studying online communities and standard processes of coding qualitative data as outlined by Carspecken [16]. The study authors (CW and MA) did all coding jointly during weekly 1-h meetings between March and September of 2020. At the start of each meeting, the principal investigator (MA) navigated to the place in each transcript in which online support was discussed by searching for the keywords "support group," "online," and "Facebook." First, we performed low-level coding by attaching describing words ("codes") to segments of the interviews. Then, we organized our codebook into the categories of the framework for studying online communities. The two primary categories, as labeled using the framework for studying online communities, included engagement and impacts. The sub-categories of engagement included initializing, responding, and keeping at a distance. The sub-categories of impacts included physical, psychological, and social domains. We discussed each excerpt until we came to consensus about which codes to apply. Once we completed coding, we identified the risks and benefits in each domain. We shared our findings with the group moderators as a form of memberchecking, and we included their insights in the final revision.

\section{Results}

A total of 40 patients were included in the study (Table 2). The mean age of study participants was 48 years old (range 30-75); 28 were female and 12 were male. At the time of the study interview (Table 2), the majority of participants were stage IV $(n=38)$. Online lung cancer support communities provide support, camaraderie, and specialized health information. Participants described engaging in the online community through (1) initializing communication through asking questions or sharing resources, (2) responding to others comments or inquiries, or (3) simply observing/reading others posts. Participation had physical, psychological, or social impacts, with benefits (e.g., empowerment) and risks (e.g., feelings of jealousy or misinformation) in each domain. Participants used various strategies to mitigate negative impacts, such as distancing oneself as needed. Participants described their experiences in online lung cancer support groups on Facebook in the context of broader conversation about coping with advanced lung cancer. 
Table 1 Examples of interview prompts

Examples of starting questions and general follow-up prompts

Share with me some about (the online support group) and some of the work that you do in there and how they are meaningful to you.

Tell me tell me everything about the support group.

Tell me about your experience with them

What is the group about?

Who are they?

What do they do?

When did you join?

How long you've been with them?

How did you join or what did you do there?

Why did you join them?

What did you find from joining them?

What do you read there?

Do you write?

Do you comment?

What else do you do?

Tell me about the work that you're doing with them, and tell me why you're doing it?

What impact are you thinking you're hoping to leave and what takes place in that group?

What the group has helped with or not helped with anything in your experience?

What do you get from joining them?

What do they mean to you?

What do you like?

What do you not like about it?

Examples of specific follow-up questions

You mentioned, "negative personality." What does that "negative personality" in this context mean?

You mentioned the "highs" and the "lows"; the good news; and the difficult news including seeing people struggle. How do you react to both of them?

You mentioned you had mixed feelings about (the online support group), share with me about that more.

We will begin by describing the findings of our first research question regarding the theme of engagement in online lung cancer support communities. Next, we describe the findings from our second research question about the psychological, social, and physical impact(s) of online engagement.

\section{Engagement}

Engagement captures the ways in which people described using the online community. While there are some similarities between how individuals seek out support in online spaces as compared with in-person (e.g., asking practical advice, offering emotional support to others) online communities provide a unique opportunity for lung cancer patients on targeted therapies to connect with others with shared experiences across geographic boundaries, to take breaks as needed, and to learn
Table 2 Participant characteristics

\begin{tabular}{ll}
\hline Participant characteristics & Median (range)/count \\
\hline Age & $49(30-75)$ years \\
Gender & 12 \\
Male & 28 \\
Female & \\
Race & 34 \\
White & 6 \\
Others (Asian, Hispanic, & \\
$\quad$ biracial (Asian and Hispanic)) & \\
Region in the USA & 18 \\
West & 8 \\
Northeast & 7 \\
Midwest & 6 \\
South & \\
Insurance & 34 \\
Private & 4 \\
Medicare & 2 \\
Medicaid & 19.5 (3-152) months \\
Time since diagnosis & \\
Cancer stage at time of interview & 38 \\
IV & 2 \\
IIIb & \\
Mutation & \\
ALK & \\
EGFR & \\
Ros1 & \\
\hline & \\
&
\end{tabular}

about others cancer journeys without necessarily having to respond. People engaged in the online lung cancer support community through initializing, responding, or keeping at a distance.

\section{Initializing}

Participants initiated engagement in several ways. People asked pragmatic questions, such as, "So I reached out to people on the ALK Facebook group and I said, What supplements are you taking? What are you eating? What are you doing? What diet are you on?" (1008). Participants also shared relevant research studies and worked to present them simply for others to digest: "I spend a lot of time reading studies and sharing the ones that I think are useful to members ... making it understandable for people who do not have a medical background. I derived a lot of pleasure from doing that" (1001). Moreover, they shared their experiences with their providers/ specialists and their management plans and reached out to others to check on them. Some simply shared uplifting, positive, and funny posts. 


\section{Responding}

Responding captured how participants reacted to other members, such as hearing other people's stories, answering questions, sharing perspectives, and amplifying experiences. Participants reciprocally exchanged information and resources to learn from others and to share what they had found helpful, e.g., "I'll ask a question. I'll try to answer somebody else's question. It's also made it seem less like I'm in the dark, like I have to do everything myself' (2013).

\section{Keeping at a distance}

Group members demonstrated varying levels of participation in the online space, including some who preferred to be less active in posting and were content with reading others' contributions, e.g., "I don't post much there but I read it, and for me that's my coping" (1007). People describing themselves as "private" took that choice. Some felt they had to distance themselves after feeling sad with others' death or cancer advancing, including taking breaks or moderating use, e.g., "There was a period where there was just a lot of deaths in the group and just kind of affecting me and I think I tried to distance myself from that a little bit, you know, just take a little break" (1003). Politics and sometimes religion were a turnoff for some and a reason for not engaging. Others were selective in their engagement, preferring not to follow every thread, especially when they did not trust all the content. Finally, some acknowledged not being as involved and wished to participate more.

\section{Impacts of engagement}

As identified in our organizing framework for studying online communities [2], we examined the potential impacts of engagement in online lung cancer support communities including the physical, psychological, and social domains. Adding on to the organizing framework, we identified the benefits and risks in each domain. We described the strategies that participants used to mitigate the risks.

\section{Physical}

Positive physical impacts included information about health management, recommendations regarding expert providers, and empowerment for self-advocacy. Participants reported gleaning information regarding their physical health. They learned important management strategies on several topics, including diagnostic pathways and treatments if cancer progressed, e.g. "I posted a question in the ROS1 group, and several ROS1 patients who were diagnosed longer than I have been and have had lots of experience were the ones who would advise not to have the whole brain radiation and to go see a ROS1 expert" (3001). Similarly important were how to deal with side effects, what diet to follow, and which supplement(s) to take. Participants received recommendations and referrals regarding expert providers. Empowered with information learned from their peers, they became better at advocating for themselves with their own providers, including conversing about new topics and bringing questions to follow-up visits.

The online space came with concerns and risks for a few participants. Some felt they could not trust all the information offered there, e.g., "There's coffee enemas and going to Mexico and apricot seeds and Laetrile. I mean, there is no end to this utter nonsense that gets perpetrated" (2003). Some expressed worries that there could be misinformation about diet, supplements, and alternative approaches. To navigate these risks, some preferred to trust their own providers and not to discuss treatment-related matters with their peers.

\section{Psychological}

Positive impacts included feelings of hope and support, such as being reassured after learning about their disease from peers and hearing what they considered inspirational stories in survivors' journeys, e.g." "It was late, and I was in my bed terrified and so alone. I put something on there. This woman in Australia, within five minutes, posted back, 'Hey, my husband's been alive for four years and going strong.' Nothing in the world could have felt better than a real human being saying to me, 'There's hope. I'm with you right now in this moment"' (1004). Receiving support from survivors who listened and understood reduced the emotional burden on family and friends.

Witnessing the ups and downs of others living with lung cancer helped normalize people's emotional struggles, e.g., "The group has helped bring it to my attention how many people there are out there with this disease. That it's more common than I thought it was. It's nice to know that there are other people out there" (1014). Connecting with others reduced feelings of social isolation, especially for those in remote areas.

Participants described feeling sad when others were not doing well, e.g., "It can get me a little depressed sometimes when I look at it and see the people that aren't doing well" (1015). They shared feeling disappointed or envious when comparing themselves to others doing better, e.g., "When the good news, when I hear people's shrinkage, you're happy for them but yeah there's some jealousy in it. I'm happy for you but I wish I can hear those words as well" (1007).

Participants described learning to distance themselves as needed, e.g., "I wouldn't give it up for anything but I do have to step back at times" (1004). They recounted learning how to foster gratitude and appreciating their circumstances (e.g., being able to work) when hearing others' bad news. 


\section{Social}

Social impacts were remarkable for participants in the sense of camaraderie and belonging to a community as well as deriving purpose from helping others. Participants were especially appreciative to have found a community of individuals that shared their experience of living with the same serious health condition. This was relevant especially to those who could not fit in other support groups or relate to others' experiences due to different natures of illnesses or ages. The sense of community gave individuals strength and connectedness. Further, many felt they were helping each other, which gave them a sense of purpose. A group member described their involvement, “. . . Hours and hours, commenting on people's posts and giving them support, checking in with them . . . It gives me a purpose and maybe a meaning of being able to help them get through this" (1011). An example is providing information for a person and their family on how to access and navigate social resources. Becoming an advocate for the community and supporting the cause of accelerating research into lung cancer treatments and improving outcomes for oncogenedriven cancers were sources of fulfillment. One member noted, "I became pretty much determined that I was going to throw myself into as much advocacy work for bringing awareness to lung cancer, doing fundraising for lung cancer events, and serving as an advocate for the cancer community" (2008).

The online support groups also brought concerns in the social domain, such as tensions around different frames of reference. For example, non-religious members often felt offended and even cynical when religious individuals would impose their views or even send prayers. One member described, "I'm sorry Facebook prayers for my scan is not for me. I mean it's my lack of that type of belief that makes me cynical about all the other things that might be offered to the group" (1012).

Many felt frustrated with individuals bringing political matters into the group or framing cancer as a political issue, e.g., "We all have lung cancer and we have better things to worry about than politics" (1009). Some complained about "negative personalities" confronting others with different attitudes toward the illness, especially those who took an excessively realistic stance and said things like "you are terminal." As some introduced themselves as lung cancer patients who never smoked, a few felt prejudice around smoking if they themselves had smoked in the past. Others missed the in-person community where they could sit with someone and eat. Finally, as they developed new friendships, they grieved their losses and often struggled as they witnessed others decline or die.

To mitigate risks, people chose not to talk about politics and to avoid negative personalities. Over time and with a sense of community/belonging, they developed ways to empathize more with others who were struggling even when the person was doing well. Some learned to celebrate successes even when they themselves were not doing very well.

\section{Discussion}

Our study provides insights about online support in an understudied population of the newest group of cancer survivors, namely lung cancer patients on targeted therapy. We identified varied patterns of engagement: initializing, responding, and keeping at a distance. Our results show that engaging in online lung cancer support communities helped individuals manage health information, receive psychological support, and find purpose in community with other patients. At the same time, engaging in online communities came with risks, such as comparison with others, feeling marginalized, or accessing misinformation. Individuals used several strategies to mitigate negative impacts. Understanding the utility of online support groups and further addressing their risks are urgent tasks, especially in the post-COVID era. These findings may help to inform recommendations for supportive care resources for lung cancer patients expanding to the online space, such as support in coping with grief and loss of community members and the use of moderators to mitigate the spread of misinformation.

Consistent with the concept of distributed health literacy [15], individuals sought specialized information and resources from other lung cancer survivors. People who share their knowledge, namely "health literacy mediators," support other community members in managing their health, becoming more active in healthcare decision-making processes, communicating with providers, and coping with a long-term condition [15]. Being among individuals in the online space with the same diagnosis fosters empathic communication through which experiences and needs are shared and understood [17]. From a communication work perspective [14], processes of exchanging messages and co-creating meaning are essential elements of the cancer experience. Connecting with peer survivors also helps mitigate the time-consuming and sometimes emotionally challenging task of managing people's questions, comments, emotions, and worries.

Our study highlights patterns of engagement in online spaces. Besides initializing and responding, keeping at a distance is particularly interesting. Remaining silent or not participating can represent heterogeneous experiences, such as a preference for reading about others' experiences (versus sharing their own), worsening health (e.g., disease progression), or competing life demands (e.g., caregiving responsibilities). Conversely, people may simply be distancing themselves in response to a community member's death, which may help to mitigate the emotional burdens of participation. Further, a less engaged person could be avoiding bad experiences, undue political debate, imposed religious views, or smoking stigmatization. The term "peripheral learners" [18] has been used to describe individuals who may be less active but are still engaged and who are perhaps observing skills and behaviors associated with active participation. Individual participation 
in online communities for lung cancer patients on targeted therapies should be considered in the context of disease progression, individual coping styles, and learning preferences [19]. Future work could longitudinally examine nuanced patterns of online engagement among individuals with lung cancer, such as the frequency of breaks and potential associations between engagement in the online space and treatment challenges.

This work's strengths include the participants own insights about their engagement with and impacts of online lung cancer support communities. We purposively sampled from three online groups to draw upon varied perspectives within the lung cancer community. However, these data represent participants' views at one time point and should be interpreted with the awareness that online lung cancer support communities can change over time due to various factors such as changes in moderator roles and responsibilities, member death, and new member engagement. Despite efforts to recruit underrepresented minorities (e.g., follow-up recruitment posts and emails emphasizing the search for people of color), this sample primarily represented privileged white upper-middle-class individuals with online access and may not be generalizable to racial/ethnic minorities. Racial and ethnic disparities persist in Internet use for health information-seeking [20] as well as in cancer incidence and outcomes more broadly in the USA [21].

\section{Conclusions}

Online lung cancer support communities are a source of specialized health information and a place of belonging. For those facing a life-threatening health condition, meeting others with the same diagnosis can provide hope through a shared understanding of the disease's psychological and physical burdens. Remaining silent may be due to various reasons, such as coping style, or to avoid negative experiences or mitigate the emotional burden. Learning strategies to adapt to challenges in the online space, such as moderating use, being selective about which threads to follow, fostering gratitude, and having empathy for others are transferable skills that prove invaluable in navigating cancer.

Acknowledgments The authors would like to thank LUNGevity, especially Upal Basu Roy, for helping connect with patient advocacy and support groups. They also thank patients and patient advocates Janet Freeman-Daily, Jill Feldman, Ivy Elkins, Rhonda Meckstroth, and Tom Carroll, for helping connect us to research participants. They also acknowledge the ROSOneder support group, the ALK-Positive Facebook Support Group, and the EGFR Resisters for supporting and promoting this work. The authors would also like to thank all study participants for contributing their time and their experiences.

Author contributions CW engaged in formal analysis, writing - original draft, writing - review and editing, and visualization. MA engaged in study conceptualization, formal analysis, writing — original draft, writing - review and editing, and visualization.

Funding Casey Walsh is supported by a National Institutes of Health/ National Cancer Institute grant (5T32 CA092408).

Data availability Interview transcripts can be made available upon reasonable request to the senior author (MA).

\section{Compliance with ethical standards}

Conflict of interest The authors declare that they have no conflict of interest.

Ethics approval This study was performed in line with the principles of the Declaration of Helsinki. The University of Washington Institutional Review Board approved the study (study number: STUDY00005438.

Consent to participate Informed consent was obtained from all individual participants included in the study.

Consent for publication The authors affirm that human research participants provided informed consent for publication.

Code availability Not applicable.

\section{References}

1. Harkin LJ, Beaver K, Dey P, Choong KA (2020) Secret groups and open forums: defining online support communities from the perspective of people affected by cancer. Digit Health 6: 2055207619898993

2. Zhang S, O'Carroll Bantum E, Owen J, Bakken S, Elhadad N (2017) Online cancer communities as informatics intervention for social support: conceptualization, characterization, and impact. J Am Med Inform Assoc 24(2):451-459

3. Gupta T, Schapira L (2018) Online communities as sources of peer support for people living with cancer: a commentary. J Oncol Pract 14(12):725-730

4. Allen C, Vassilev I, Kennedy A, Rogers A (2016) Long-term condition self-management support in online communities: a metasynthesis of qualitative papers. J Med Internet Res 18(3):e61

5. van Eenbergen MC, van de Poll-Franse LV, Heine P, Mols F (2017) The impact of participation in online cancer communities on patient reported outcomes: systematic review. JMIR Cancer 3(2):e15

6. Brown Johnson CG, Brodsky JL, Cataldo JK (2014) Lung cancer stigma, anxiety, depression, and quality of life. J Psychosoc Oncol 32(1):59-73

7. Lebel S, Devins GM (2008) Stigma in cancer patients whose behavior may have contributed to their disease. Future Oncol 4(5): 717-733

8. Centers for Disease Control and Prevention (2020) An update on cancer deaths in the United States. US Department of Health and Human Services, Centers for Disease Control and Prevention, Division of Cancer Prevention and Control, Atlanta

9. Yuan M, Huang L-L, Chen J-H, Wu J, Xu Q (2019) The emerging treatment landscape of targeted therapy in non-small-cell lung cancer. Signal Transduct Target Ther 4(1):61

10. Al Achkar M, Marchand L, Thompson M, Chow LQM, Revere D, Baldwin LM (2020) Unmet needs and opportunities for improving 
care for patients with advanced lung cancer on targeted therapies: a qualitative study. BMJ Open 10(3):e032639

11. Richards M, Anderson M, Carter P, Ebert BL, Mossialos E (2020) The impact of the COVID-19 pandemic on cancer care. Nat Can 1(6):565-567

12. McAlpine H, Joubert L, Martin-Sanchez F, Merolli M, Drummond KJ (2015) A systematic review of types and efficacy of online interventions for cancer patients. Patient Educ Couns 98(3):283-295

13. Taylor J, Pagliari C (2019) The social dynamics of lung cancer talk on Twitter, Facebook and Macmillan.org.uk. NPJ Digit Med 2:51

14. Donovan-Kicken E, Tollison AC, Goins ES (2012) The nature of communication work during cancer: advancing the theory of illness trajectories. Health Commun 27(7):641-652

15. Edwards M, Wood F, Davies M, Edwards A (2015) 'Distributed health literacy': longitudinal qualitative analysis of the roles of health literacy mediators and social networks of people living with a long-term health condition. Health Expect 18(5):1180-1193

16. Carspecken PF (1996) Critical ethnography in educational research: a theoretical and practical guide. Routledge, New York
17. Hargreaves S, Bath PA, Duffin S, Ellis J (2018) Sharing and empathy in digital spaces: qualitative study of online health forums for breast cancer and motor neuron disease (Amyotrophic Lateral Sclerosis). J Med Internet Res 20(6):e222

18. Honeychurch S, Bozkurt A, Singh L, Koutrapoulos A (2017) Learners on the periphery: lurkers as invisible learners. EURODL 20(1):191-211

19. Badreddine BM, Blount $Y$ (2019) Understanding influential factors behind lurking behaviour in online cancer communities. Behav Inform Technol. https://doi.org/10.1080/0144929X.2019.1709545

20. Laz TH, Berenson AB (2013) Racial and ethnic disparities in internet use for seeking health information among young women. J Health Commun 18(2):250-260

21. Zavala VA, Bracci PM, Carethers JM et al (2020) Cancer health disparities in racial/ethnic minorities in the United States. Br J Cancer. https://doi.org/10.1038/s41416-020-01038-6

Publisher's note Springer Nature remains neutral with regard to jurisdictional claims in published maps and institutional affiliations. 